\title{
Isoflavone production in Cyclopia subternata Vogel (honeybush) suspension cultures grown in shake flasks and stirred-tank bioreactor
}

\author{
Adam Kokotkiewicz • Maria Luczkiewicz • Wiktor Kowalski • \\ Anna Badura • Natalia Piekus • Adam Bucinski
}

Received: 28 March 2013 /Revised: 14 June 2013 / Accepted: 4 July 2013 / Published online: 20 July 2013

(C) The Author(s) 2013. This article is published with open access at Springerlink.com

\begin{abstract}
Suspension cultures of the endemic South-African plant Cyclopia subternata were established for the first time and evaluated for the presence of isoflavones. The influence of light, as well as medium supplementation strategies with phenylalanine, casein hydrolysate and coconut water on biomass growth and isoflavone production were examined. The highest levels of 7-O- $\beta$-glucosides of calycosin, pseudobaptigenin and formononetin $(275.57,125.37$ and $147.28 \mathrm{mg} / 100 \mathrm{~g} \mathrm{DW}$, respectively) were recorded for cultures grown in the absence of light, whereas coconut water substantially promoted biomass growth. Cell suspensions were subsequently grown in the 2-1 stirred-tank bioreactor. Maximum productivity of $7-O-\beta$-glucosides of calycosin, pseudobaptigenin and formononetin $\left(0.96,0.44\right.$ and $0.22 \mathrm{mg} \mathrm{l}^{-1}$ day $^{-1}$, respectively) in bioreactorcultivated cells was obtained for biomass grown in the dark and supplemented with coconut water. The results indicate that $C$. subternata suspension cultures can be utilised for the production of the specified isoflavone derivatives absent in the intact plant.
\end{abstract}

Keywords Cyclopia subternata · Cell suspension cultures . Isoflavones $\cdot$ Stirred-tank bioreactor $\cdot$ Upstream processing

A. Kokotkiewicz $\cdot$ M. Luczkiewicz $(\square) \cdot$ W. Kowalski The Chair and Department of Pharmacognosy, Faculty of Pharmacy, Medical University of Gdansk, al. gen. J. Hallera 107, 80-416 Gdansk, Poland

e-mail: mlucz@gumed.edu.pl

A. Badura $\cdot$ N. Piekus $\cdot$ A. Bucinski

Department of Biopharmacy, Faculty of Pharmacy, Ludwik Rydygier Collegium Medicum in Bydgoszcz, Nicolaus Copernicus University in Torun, ul. dr A. Jurasza 2, 85-089 Bydgoszcz, Poland

\section{Introduction}

Isoflavones are among the most extensively studied groups of natural compounds of polyphenolic character, which is mostly due to their affinity to estrogen $\beta$ receptors in humans. Isoflavones are claimed to exhibit numerous health-promoting effects, including the alleviation of menopausal symptoms, the prevention of osteoporosis and cardiovascular diseases, and lowering the risk of breast cancer (Patisaul and Jefferson 2010). The growing demand for isoflavonoid derivatives resulted in numerous research projects focused on the in vitro cultures of selected plant species, mostly from Fabaceae family. Studies dealing with biotechnological production of isoflavones are mainly based on suspension cultures of Pueraria sp. (Li and Zhang 2006; Goyal and Ramawat 2008a, b; Sharma et al. 2009), Psoralea sp. (Shinde et al. 2009a), Glycine max (Federici et al. 2003; Terrier et al. 2007) and Genista tinctoria (Luczkiewicz and Glod 2005; Luczkiewicz and Kokotkiewicz 2012). These are characterised mainly by the presence of genistein, genistin, daidzein and puerarin, which belong to the best known phytoestrogens (Patisaul and Jefferson 2010). On the other hand, limited data are available concerning the utilisation of largescale plant in vitro systems for the production of methoxy- or methylenedioxyisoflavones, such as calycosin, formononetin, pseudobaptigenin and their respective glucosides. Natural resources of Astragalus membranaceus, considered a major source of free and glucosidated calycosin and formononetin, are quite rare due to its limited distribution and relatively slow growth (Wu et al. 2011), and are also characterised by varying isoflavone content (Wu et al. 2005). Calycosin, formononetin and pseudobaptigenin are also present in the more widespread legume Trifolium pratense; however, their isolation would require preparative separation of a rather complex set of ca. 30 isoflavones (Wu et al. 2003).

Establishing a biotechnological source of the above derivatives may be of interest, as they exhibit numerous biological 
properties which make them potentially useful for medical applications. For instance, calycosin possesses distinct estrogenic properties (Chen et al. 2011), whereas its 7-O- $\beta$ glucoside was shown to alleviate pathologic changes in rabbit model of osteoarthritis (Choi et al. 2007). Another methoxylated isoflavone, formononetin, proved to inhibit bone loss in ovariectomised rats (Ha et al. 2010) and exert positive effects on the metabolism of human osteoarthritic osteoblasts (Huh et al. 2010). Pseudobaptigenin exhibits antiproliferative effects on selected human stomach and colon cancer cell lines (Yanagihara et al. 1993) and recent bioinformatics study showed that it may act as protein tyrosine kinase inhibitor (Hashemi et al. 2012).

Our research into the polyphenolic composition of in vitro cultures of the endemic South-African plant Cyclopia subternata (honeybush) showed that its callus cultures are characterised by the presence of isoflavone derivatives absent in the intact plant material, namely 7-O- $\beta$-glucosides of calycosin (CG), pseudobaptigenin (PG) and formononetin (FG) (Kokotkiewicz et al. 2012b). However, biotechnological production of secondary metabolites in plant cell cultures requires the use of liquid media, which provide superior conditions for bioprocess optimization, increasing system productivity and scaling-up with the use of bioreactors (Georgiev et al. 2009, 2013). Thus, the aim of the research was to establish an in vitro source of biologically active methoxylated isoflavones based on cell cultures of $C$. subternata. Suspension cultures were to be initiated and examined for the presence of polyphenolic compounds. In the next part of the study, the effects of light regime and medium supplementation strategies on isoflavone production were to be evaluated. Finally, the cell cultures were adapted to bioreactor conditions in order to determine the effect of the scale-up and the altered mixing and aeration patterns on biomass growth and polyphenol content. To our knowledge, this is the first report dealing with the biosynthesis of isoflavone derivatives in suspension cultures of Cyclopia plants. At the same time, the presented study is one of the very few approaches oriented towards the production of methoxylated isoflavones with the use of plant in vitro cultures.

\section{Materials and methods}

Plant material and culture conditions

The previously obtained C. subternata callus of friable consistency, grown on the modified Murashige and Skoog (MS) medium (hereafter referred to as the $\mathrm{MS}_{\mathrm{C}}$ medium) supplemented with $20.19 \mu \mathrm{MN} N$-(2-chloro-4-pirydyl)- $N$ 'phenulurea (4-CPPU) and $1.96 \mu \mathrm{M} 2,4,5$-trichlorophenoxyacetic acid (2,4,5-T) (Kokotkiewicz et al. 2009), was used for the initiation of suspension cultures. The above strain is deposited in the Higher Plants Biotechnology Laboratory, Department of Pharmacognosy, Medical University of Gdansk, Poland, and is available to other scientists via scientific collaboration. C. subternata callus was maintained by subculturing onto the fresh medium every 20 days. The details on medium preparation were described before (Kokotkiewicz et al. 2009). All experiments were conducted in the growth chamber at $24 \pm 1{ }^{\circ} \mathrm{C}$. Unless otherwise stated, the cultures were grown under continuous light $\left(88 \pm 8 \mu \mathrm{mol} \mathrm{m}{ }^{-2} \mathrm{~s}^{-1}\right.$, Philips TLD fluorescent tubes $36 \mathrm{~W}$ ).

Preparation of coconut water, casein hydrolysate and phenylalanine feedstocks

Coconut water $(\mathrm{CW})$, casein hydrolysate $(\mathrm{CH})$ and phenylalanine (PA) were of plant cell culture grade (Sigma-Aldrich, St. Louis, MO, USA). CW was available as deproteinised and sterile filtered product ( $\mathrm{pH} 4.5-5.7$, osmolality 287 $487 \mathrm{mOsm} / \mathrm{kg}$ water according to the specification) and kept at $-20{ }^{\circ} \mathrm{C}$ prior to use. After bringing to room temperature, the residual protein precipitate was allowed to settle and removed via decantation. The so prepared $\mathrm{CW}$ feedstock was added to the growth medium without further filtration. The 2.0- $\mathrm{mg} \mathrm{ml}^{-1}$ stock aqueous solutions of $\mathrm{CH}$ and PA were sterile-filtered through $0.2 \mu \mathrm{m}$ nylon filters (SUN-Sri, Rockwood, TN, USA).

Suspension cultures grown in shake flasks

For the initiation of suspension cultures, $1.25 \mathrm{~g}$ portions of friable C. subternata callus (taken on 20th day of the growth cycle) were inoculated into 125 -ml Erlenmeyer flasks containing $50.0 \mathrm{ml}$ of liquid $\mathrm{MS}_{\mathrm{C}}$ medium, closed with silicone foam stoppers (Carl-Roth, Karlsruhe, Germany) and grown on an orbital shaker (120 rpm, $25.4 \mathrm{~mm}$ stroke, INNOVA 2350, New Brunswick Scientific, Enfield, CT, USA) under continuous light. Biomasses and the respective media were collected up to 40th day in 2-day intervals. The experiment was repeated in triplicate (63 flasks in total, 3 samples per day). In a parallel experiment, conducted in the same manner as described above, the suspension cultures were grown in complete darkness ( 63 flasks in total, 3 samples per day).

For the feeding experiments, the suspension cultures were initiated as described above and cultivated under continuous light. On the 16 th day, the growth medium was supplemented with $\mathrm{CW}(5 \% v / v, 2.5 \mathrm{ml}$ of $\mathrm{CW}$ feedstock per flask), casein hydrolysate ( $100 \mathrm{mg} \mathrm{l}^{-1}, 2.5 \mathrm{ml}$ of stock solution per flask) or L-phenylalanine (100 mg $\mathrm{l}^{-1}, 2.5 \mathrm{ml}$ of stock solution per flask). 


\section{Bioreactor experiments}

The stirred-tank bioreactor (STB) system used in the experiments consisted of the $31(170 \times 300 \mathrm{~mm})$ glass BioProbe ${ }^{\mathrm{TM}}$ vessel equipped with plate type $(76 \times 108 \mathrm{~mm})$, magnetically driven impeller (Bellco Glass, Vineland, NJ, USA), Variomag Biosystem Direct magnetic stirrer (Thermo Fisher Scientific, Waltham, MA, USA), dissolved oxygen controller (Cole-Parmer, Vernon Hills, IL, USA), Optima air pump (Hagen, Montreal, QC, Canada), glass wool prefilter, flowmeter, air humidifier, inlet and outlet air sterilisation filters (PTFE membrane, $0.2 \mu \mathrm{m}$ pore size, Cole-Parmer) and sintered glass sparger. Schematic diagram of the bioreactor is presented in Fig. 1.

For the experiment, 50.0-g portions of friable parenchymatic callus were inoculated into the culture vessel containing 2,000 $\mathrm{ml}$ (working volume) of $\mathrm{MS}_{\mathrm{C}}$ medium. The mixing speed was set at $40 \mathrm{rpm}$, and air saturation was held at $50 \%$ (at 0.2 vvm air flow rate). Biomass and medium samples were collected on 4th, 8th, 12th, 16th, 20th, 24th, 28th, 32 nd and 40th days by unloading the bioreactor in whole (batch cultivation). The experiment was repeated twice.

In the modified experiment, the cell suspension was grown without the presence of light in the $\mathrm{MS}_{\mathrm{C}}$ medium supplemented with $0.1 \% v / v$ SE-15 antifoam (SigmaAldrich) prior to autoclaving. The culture was additionally supplemented with $5 \% v / v$ CW (Sigma-Aldrich) on the 16 th day (100 $\mathrm{ml}$ of CW feedstock per bioreactor). Biomass and medium samples were collected on the 16th (prior to the addition of $\mathrm{CW}$ ), 20th, 24th, 28th and 32nd days by unloading the bioreactor in whole. The experiment was conducted in duplicate.

\section{Determination of cell biomass}

Biomass samples harvested in the course of experiments were separated from the media with the use of vacuum filtration assembly (G4 glass frit funnel). The respective media samples were retained and stored at $-20{ }^{\circ} \mathrm{C}$ prior to analysis. The collected biomasses were freeze-dried with the use of LYOVAC GT2 apparatus (Finn-Aqua SantasoloSohlberg, Tuusula, Finland), weighed-dry weight (DW) determination - and subjected to phytochemical analysis.

Evaluation of polyphenol content

Qualitative and quantitative analyses of polyphenolic compounds in freeze-dried biomass samples followed the extraction procedure and high-performance liquid chromatography (HPLC) method described before (Kokotkiewicz et al. 2012a). The chromatographic system used (Shimadzu, Kyoto, Japan) consisted of two solvent pumps (LC-20 AD), a liquid phase degasser (DGU-20A3), an autosampler (SIL$20 \mathrm{AC}, 24{ }^{\circ} \mathrm{C}$ ), a column oven (CTO-20 AC, $30{ }^{\circ} \mathrm{C}$ ), a diode array detector (SPD-M20A) and a mass spectrometry detector (2010EV). Separations were performed on Supelcosil LC-18 column $(150 \times 4.6 \mathrm{~mm}, 3 \mu \mathrm{m}$ particle size, Sigma-Aldrich $)$ at $0.6 \mathrm{ml} \mathrm{min}{ }^{-1}$ flow rate. The mobile phase consisted of $\mathrm{A}$ : water/formic acid $(1000: 1 \mathrm{v} / \mathrm{v})$ and $\mathrm{B}$, water/acetonitrile/ formic acid (500:500:1 v/v/v). The gradient program was as follows - 0-15 min, 12-25\% B; 15-75 min, 25-100\% B; 75$77 \mathrm{~min}, 100 \% \mathrm{~B}$; 77-80 min, 100-12\% B; $82 \mathrm{~min}$, stop. The injection volume was $3 \mu \mathrm{l}$. The LC-DAD data were recorded over the 190-800 $\mathrm{nm}$ range, and the $280 \mathrm{~nm}$ wavelength was used for quantitative analyses. MS spectra were acquired in negative ion mode over the range of $\mathrm{m} / \mathrm{z} 50-1800$, using
Fig. 1 Schematic diagram of the stirred-tank bioreactor: 1 culture vessel, $1 a$ inoculation port, $1 b$ plate impeller, $l c$ glass frit sparger, 2 magnetic stirrer, 3 dissolved oxygen probe, 4 dissolved oxygen controller, 5 air pump, 6 air prefilter, 7

flowmeter, 8 air humidifier, 9,10 air sterilisation filters. Arrows indicate air flow direction

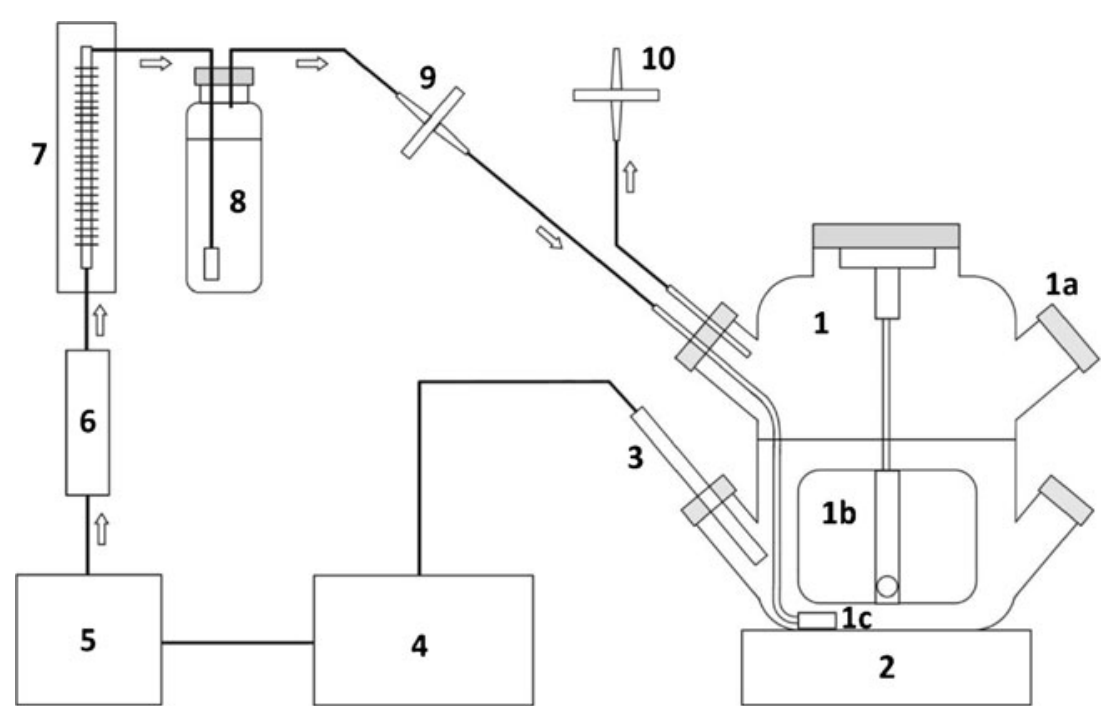


scan acquisition mode. The following ESI parameters were applied - desolvation at $230{ }^{\circ} \mathrm{C}, 2,000 \mathrm{~V}$ interface voltage, $2,000 \mathrm{~V}$ detector voltage, nebulising gas flow at $1.51 \mathrm{~min}^{-1}$, drying gas flow at $15.01 \mathrm{~min}^{-1}$. The data were processed with LC-MS solution 3.40 software (Kyoto, Japan).

Media samples were brought to room temperature, vortexed for $1 \mathrm{~min}$ at $1,000 \mathrm{rpm}$, centrifuged at 7,500 $\times \mathrm{g}$ for $15 \mathrm{~min}$ and subjected to HPLC analysis.

\section{Statistical analysis}

The data showing the effects of different experimental strategies on biomass growth and isoflavone concentrations were subjected to one-way analysis of variance followed by Tukey's post hoc test. Values marked with different letters were considered to be statistical different at $p<0.05$. The data were analyzed using Statistica software (StatSoft, Tulsa, OK, USA).

\section{Results}

The effect of lighting conditions on biomass growth and isoflavone content in suspension cultures grown in shake flasks

As shown in Fig. 2a, the recorded DW values were similar in the case of darkened and irradiated cultures, and there were no significant differences in the growth kinetics between the two experiments. The growth profiles were characterised by well-defined expotential phase, followed by short stationary phase and subsequent decline (Fig. 2a). The suspension retained bright yellow colour and vital appearance until the $32^{\text {nd }}$ day of the experiment, when necrotic changes (browning and decrease in cell hydration) became visible.

C. subternata suspension cultures were characterised by the presence of isoflavone glucosides (CG, PG and FG) and the absence of both xanthone (mangiferin and isomangiferin) and flavanone (hesperidin) derivatives. The production curves of isoflavones overlapped each other and showed clearly marked maxima followed by rapid decline (Fig. 2b-d). Maximum concentrations of CG, PG and FG obtained in the case of irradiated cultures $(148.34,62.54$ and $99.21 \mathrm{mg} / 100 \mathrm{~g}$ DW, respectively) were substantially lower than in cell suspension grown without the presence of light $(275.57,125.37$ and $147.28 \mathrm{mg} / 100 \mathrm{~g} \mathrm{DW}$, respectively). The production maxima in darkened cultures were also delayed in comparison to cells grown under continuous light.

Regardless of the lighting conditions applied, isoflavone concentrations achieved in cell suspension were significantly higher than in the inoculation callus biomass $(7.52,7.19$ and $6.44 \mathrm{mg} / 100 \mathrm{~g}$ DW for CG, PG and FG, respectively, represented as day 0 in Fig. $2 b-d$ ). As revealed by HPLC, none of the metabolites examined was excreted into the growth medium.

The effect of precursor feeding and nutrient supplementation on biomass growth and isoflavone content in suspension cultures grown in shake flasks

As shown in Fig. 3a, CW-supplemented suspension cultures entered stationary phase on the 20th day of experiment, i.e. 6 days earlier than the control group. Moreover, the amounts of DW obtained (ca. $16 \mathrm{~g} \mathrm{l}^{-1}$ ) were substantially higher in comparison to control and stayed at the same level until the 32nd day of the experiment (Fig. 3a). The production curves of isoflavones in CW-fed cultures were characterised by two distinct maxima, corresponding to the early and late stationary phases (Fig. 3b-d). As a result, productivity of isoflavone derivatives (with exception of FG) was substantially elevated in suspension cultures supplemented with coconut water (Table 1).

On the contrary, $\mathrm{CH}$ and PA supplementation had no significant effect on cell growth (Fig. 3a). The moderate effect on isoflavone biosynthesis was manifested by the additional production maximum on the day 28th (Fig. 3b-d). However, the obtained system productivity remained lower in comparison to suspension culture supplemented with $\mathrm{CW}$ (Table 1). Similarly to the control culture, no isoflavones were released into the growth media.

\section{Bioreactor experiments}

The growth kinetics of bioreactor-grown C. subternata cultures were similar to those of biomass cultivated in shake flasks (Figs. 3a and 4a), except that the stationary phase was more evident in the case of bioreactor culture (Fig. 4a). STBgrown culture also showed substantial foaming and wallgrowth, resulting in lumps of aggregated material. The suspension, however, retained vitality until the 32nd day of culture. No volume loss was recorded during the 40-day cultivation period.

Production curves of isoflavones in STB-grown biomass were characterised by two distinct maxima, corresponding to expotential and late stationary phase, respectively (Fig. 4b-d). The maximum concentrations of CG and PG (154.03 and $66.11 \mathrm{mg} / 100 \mathrm{~g} \mathrm{DW}$, respectively) were comparable to culture-grown in shake flasks, whereas FG concentration $(40.28 \mathrm{mg} / 100 \mathrm{~g} \mathrm{DW})$ was lower.

In the modified experiment, $C$. subternata suspension was grown without the presence of light and additionally supplemented with an antifoam agent (day 0) and CW (day 16). The biomass was harvested since the middle expotential phase (day 16) until the 32nd day. Earlier stages of the culture (low biomass yield), as well as the 40th day (decline), were omitted. 

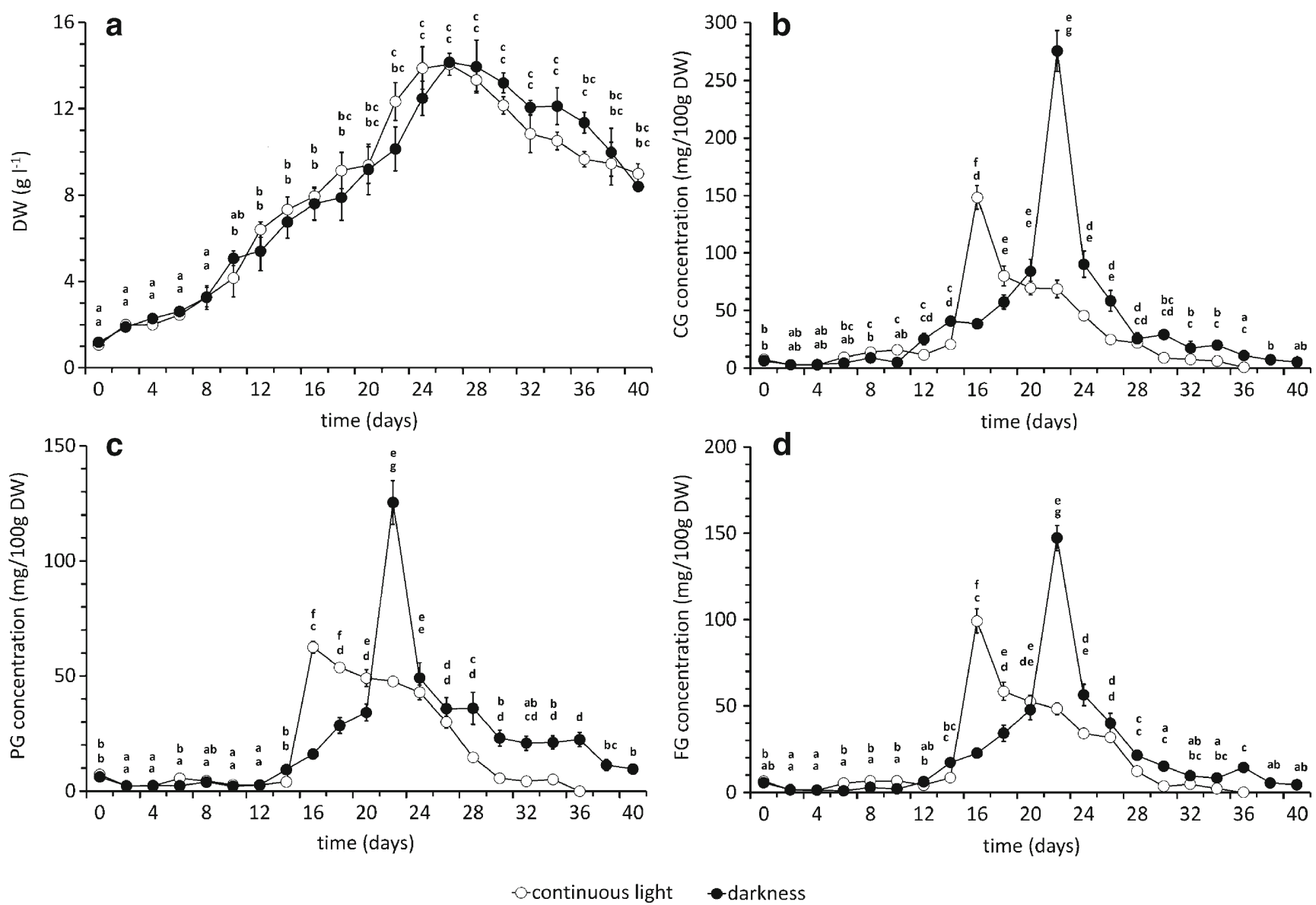

Fig. 2 Changes in DW (dry weight) (a) and concentrations of 7-O- $\beta$ glucosides of calycosin (b), pseudobaptigenin (c) and formononetin (d) in shake flask-grown C. subternata suspension cultured under continuous light or in the dark. Different letters indicate significant

differences between means $(n=3)$ based on Tukey's range test $(p<0.05)$. Letters arranged in columns refer to the values obtained for illuminated (top) and darkened (bottom) cultures on the respective days of the experiment

As in the case of suspension grown in shake flasks, maximum biomass yield in $\mathrm{CW}$-supplemented culture was achieved faster than in control group (Fig. 4a). The changes in isoflavone metabolism were also evident-only one production maximum was recorded, which also occurred later than in the culture grown under continuous light. The obtained isoflavone concentrations (195.53, 90.19 and $45.48 \mathrm{mg} / 100 \mathrm{~g} \mathrm{DW}$ for CG, PG and FG, respectively) were higher than in control culture (Fig. 4b-d). The examined secondary metabolites were stored intracellularly.

\section{Discussion}

Establishing the suspension cultures

Cell suspension cultures of $C$. subternata were initiated by mechanical dispersion of C. subternata callus in the liquid MS medium. Since the friable and loose consistency of the initial biomass was related to rather high concentration
(20.19 $\mu \mathrm{M}$ ) of 4-CPPU (Kokotkiewicz et al. 2009), liquid medium of the same composition (referred to as $\mathrm{MS}_{\mathrm{C}}$ ) was used in current experiments. As a result, homogenous suspension culture was obtained. The observed effect of 4CPPU on cytodifferentiation in C. subternata in vitro cultures is consistent with the previous reports, which indicated that, when applied at $\geq 2.5 \mu \mathrm{M}$ concentration, it induces callus formation in selected legumes, such as Phaseolus vulgaris and Vicia faba (Mohamed et al. 1992).

Similarly to the previously described callus cultures (Kokotkiewicz et al. 2009), C. subternata suspension showed no presence of xanthone derivatives, i.e. mangiferin and isomangiferin, which are major phenolic constituents of the intact plant (De Beer and Joubert 2010). These results are contrary to other reports on suspension cultures of xanthoneproducing plants from the genera Centaurium (Beerhues and Berger 1994) and Hypericum (Pasqua et al. 2003), which retained the ability of xanthone biosynthesis. The established C. subternata suspension cultures also did not accumulate the flavanone hesperidin, present in substantial quantities 

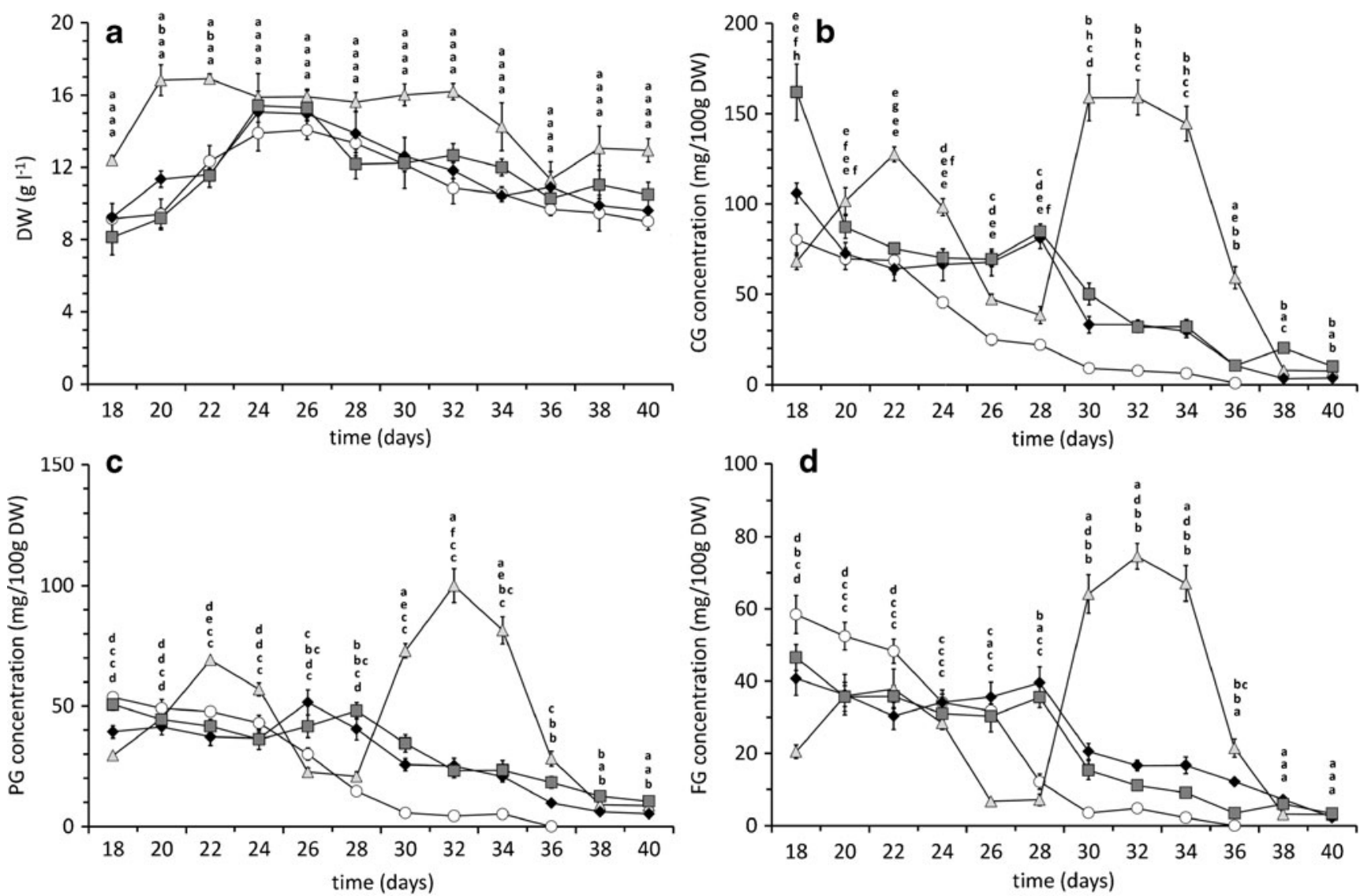

-control $九 \mathrm{CW} \leftarrow \mathrm{CH} \rightarrow \mathrm{PA}$

Fig. 3 Changes in DW (dry weight) (a) and concentrations of 7-O- $\beta$ glucosides of calycosin (b), pseudobaptigenin (c) and formononetin (d) in shake flask-grown C. subternata suspension, cultured without supplementation (control) or supplemented with $5 \% v / v$ coconut water (CW), $100 \mathrm{mg} \mathrm{l}^{-1}$ casein hydrolysate $(\mathrm{CH})$ or $100 \mathrm{mg} \mathrm{l}^{-1}$ phenylalanine (PA) on the $16^{\text {th }}$ day. All cultures were grown under continuous light.

in the intact plant (De Beer and Joubert 2010). Similar phenomenon was observed in unorganized biomasses of Citrus sinensis and Citrus paradisi, which gradually almost
Different letters indicate significant differences between means $(n=3)$ based on Tukey's range test $(p<0.05)$. Letters arranged in columns refer to the values obtained for control and cultures supplemented with $\mathrm{CW}$, $\mathrm{CH}$ and PA (from top to bottom, respectively) on subsequent days of the experiment

completely lost the ability to biosynthesize naringin, i.e. the flavanone derivative abundant in the mother plant (Barthe et al. 1987). Even though these constituents were not found in

Table 1 Maximum productivities of calycosin 7-O- $\beta$-glucoside (CG), pseudobaptigenin 7-O- $\beta$-glucoside (PG) and formononetin 7- $O$ - $\beta$-glucoside (FG) in C. subternata suspension cultures grown under different experimental conditions

\begin{tabular}{llllr}
\hline Experimental conditions & Day of the culture & \multicolumn{2}{l}{ Productivity $\left(\mathrm{mg} \mathrm{l}^{-1} \mathrm{~d}^{-1}\right)$} \\
\cline { 3 - 5 } & & CG & PG & FG \\
\hline Shake flasks (continuous light) & 16 & 0.74 & 0.31 & 0.49 \\
Shake flasks (darkness) & 22 & 1.27 & 0.58 & 0.68 \\
Shake flasks (continuous light+CW) & 22 & 0.98 & 0.53 & 0.29 \\
Shake flasks (continuous light+CH) & 18 & 0.54 & 0.20 & 0.21 \\
Shake flasks (continuous light+PA) & 18 & 0.73 & 0.23 & 0.21 \\
STB (continuous light) & 20 & 0.70 & 0.30 & 0.18 \\
STB (darkness, AF+CW) & 28 & 0.96 & 0.44 & 0.18 \\
\end{tabular}

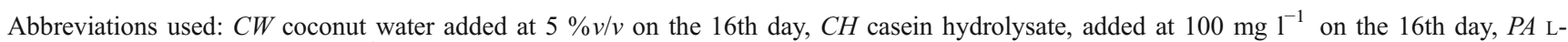
phenylalanine added at $100 \mathrm{mg} \mathrm{l}^{-1}$ on the 16th day, STB stirred-tank bioreactor, $A F$ SE- 15 antifoam added at $0.1 \% v / v$ on the inoculation day 

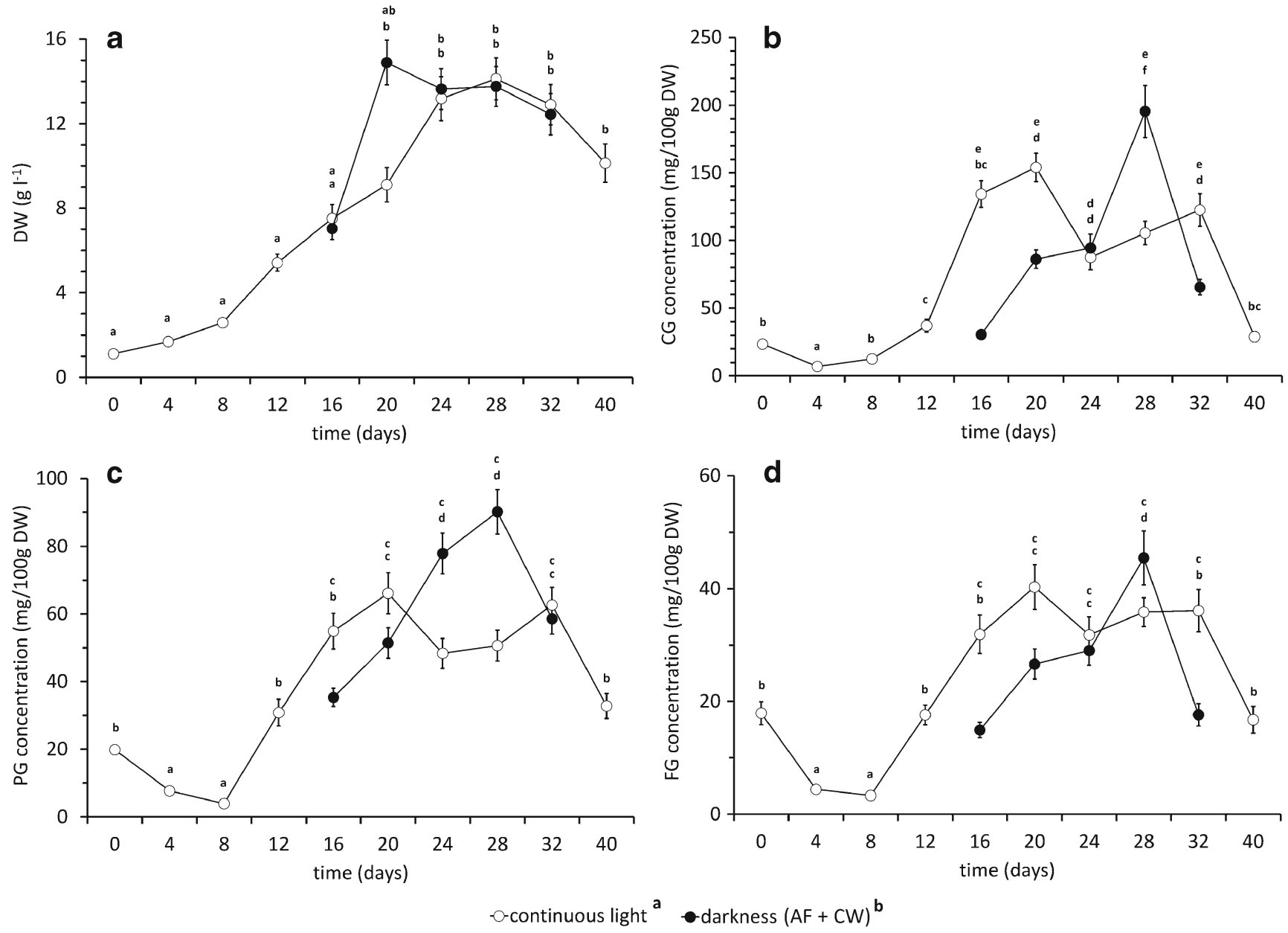

Fig. 4 Changes in DW (dry weight) (a) and concentrations of 7-O- $\beta$ glucosides of calycosin (b), pseudobaptigenin (c) and formononetin (d) in C. subternata suspension grown in stirred-tank bioreactor under different experimental conditions. Different letters indicate significant differences between means $(n=2)$ based on Tukey's range test $(p<0.05)$. Letters arranged in columns refer to the values obtained for illuminated (top) and darkened (bottom) cultures on the respective days of the experiment.

a Cell suspension cultured without additional supplementation, harvested until day 40th in 4-day intervals; $\mathbf{b}$ cell suspension cultured in the presence of $0.1 \%$ SE-15 antifoam (added on the inoculation day) and $5 \% v / v$ coconut water (added on the 16th day) and harvested on the following days: 16, 20, 24, 28 and 32. The culture on the day 16 of the experiment was harvested prior to $\mathrm{CW}$ supplementation

C. subternata suspension, it produced a set of isoflavone glucosides (CG, PG and FG), which were formerly found in callus of this species but not in the intact plant material (Kokotkiewicz et al. 2012b). No isoflavones were detected in the growth medium, reflecting the typical, intracellular storage profile of the above compounds (Goyal and Ramawat 2008a, b; Sharma et al. 2009; Shinde et al. 2009a; Luczkiewicz and Kokotkiewicz 2012).

The presented results, together with the outcome of former reports (Kokotkiewicz et al. 2012a, b), confirm that the biosynthesis of polyphenols in Cyclopia in vitro cultures depends on both compound type and biomass cytodifferentiation. Moreover, it is worth noting that, due to the complete absence of hesperidin, a flavanone present in intact plant and callus cultures (Kokotkiewicz et al. 2009), the suspension biomass constitutes a selective, new source of the examined isoflavones.
The effect of lighting conditions on biomass growth and isoflavone content

Light regime proved to exhibit substantial effect on isoflavone metabolism in legume plants. The presence of light seems to be beneficial but not necessary for the biosynthesis of the above compounds. For instance, G. tinctoria callus growing in complete darkness accumulated large amounts of genistein derivatives, but their content in irradiated cultures was even higher. On the other hand, absence of light selectively influenced the production of some isoflavones, such as malonyl- and acetylesters of genistein in G. tinctoria callus and kievitone in P. vulgaris cotyledons (Luczkiewicz 2008). Considering the above, it was of interest to evaluate the influence of light on growth and secondary metabolite biosynthesis in C. subternata suspension cultures. 
As can be seen in Fig. 1a, the lighting conditions practically did not influence the growth of the examined culture. This was expectable as parenchymatic cultures are rarely characterised by photoautotrophic growth. The highest isoflavone concentrations in both experiments were recorded during expotential growth and rapidly declined as the biomass entered the stationary phase (Fig. 2b-d). The decrease in the glucosides (CG, PG and FG) content was not accompanied by an increase in the concentrations of the respective aglycones, which suggests that the above compounds were shifted to other metabolic pathways. The observed production pattern is quite uncommon, as in most studies on cell suspension cultures of legume plants the accumulation of major isoflavonoid constituents is directly proportional to cell growth, with maximum levels achieved at the beginning of stationary phase (Federici et al. 2003; Luczkiewicz and Glod 2005; Boonsnongcheep et al. 2010; Karwasara and Dixit 2012). Another unexpected result was that CG, PG and FG concentrations were substantially higher in the cultures grown in complete darkness (Fig. 2b-d), whereas the presence of light is generally considered a stimulating factor in flavonoid biosynthesis (Luczkiewicz 2008). One possible explanation of the above phenomenon is that the lack of light acted as a stress factor triggering the production of phytoalexins. Although no experiments concerning the influence of lighting conditions on the biosynthesis of the examined compounds have been conducted before, it was shown that the stress factor in the form of low temperature stimulates the production of calycosin and its 7-O- $\beta$-glucoside in $A$. membranaceus tissues (Pan et al. 2007). This may support the hypothesis that higher isoflavone concentrations in $C$. subternata suspension grown without the presence of light are related to environmental stress. However, the observed phenomenon cannot be explained without the appropriate enzymatic studies, which would reveal the influence of light irradiation on the activities of the respective enzymes and expression of genes related to phenylpropanoid pathway.

The effect of precursor feeding and nutrient supplementation on biomass growth and isoflavone content

Although isoflavonoid content of the examined cell suspension was substantially elevated in comparison to the inoculation callus culture, rapid decline in concentrations of CG, PG and FG prior to stationary phase was unfavourable in terms of system productivity. A similar phenomenon was observed in the case of suspension cultures of G. $\max$, and it was suggested that sugar residues of isoflavone glucosides might be utilised as a carbon source by the fast growing biomass (Gueven and Knorr 2011). Therefore, the medium was supplemented with organic nutrients $(\mathrm{CW}$ and $\mathrm{CH})$ on the 16th day in order to stimulate cell growth and isoflavone biosynthesis and/or to prevent the loss of already produced glucoside derivatives. The precursor of flavonoid biosynthesis (PA) was also included in the experiments. The above substances were previously shown to effectively stimulate polyphenol production and/or biomass growth in numerous in vitro systems of higher plants. For instance, $\mathrm{CH}$ proved to be effective in the case of Pueraria lobata suspension cultures, increasing total isoflavone yield by ca. $40 \%$ ( $\mathrm{Li}$ and Zhang 2006). In Psoralea corylifolia hairy root cultures, supplementation with phenylalanine resulted in $30 \%$ increase in genistein and daidzein levels (Shinde et al. 2009b). Coconut water stimulated anthocyanin production in the callus of Rudbeckia hirta, increasing the total pigment content by over $60 \%$ (Luczkiewicz and Cisowski 2001).

In our experiments, $\mathrm{CW}$ proved to be most effective in terms of stimulating biomass growth and isoflavone productivity. As seen in Fig. 3, CW-supplemented cultures were characterised by extended stationary phase and two distinct maxima in profiles of isoflavone accumulation. The second maximum, which was not recorded in the case of control culture, may result from de novo biosynthesis during the plateau phase. This shows that isoflavone production in $C$. subternata suspension may not be restricted to intensively dividing cells, as it could be inferred from experiments on unsupplemented cultures (Fig. 1). A similar phenomenon, related to the biosynthesis of genistein and daidzein, was previously observed during the work with cell cultures of $G$. tinctoria (Luczkiewicz and Glod 2005).

The effectiveness of $\mathrm{CW}$ regarding the improvement of isoflavone productivity is most likely attributed to its high carbohydrate content (up to $5.2 \% \mathrm{w} / \mathrm{w}$ according to Yong et al. 2009), as sucrose feeding was previously shown to stimulate isoflavone biosynthesis and biomass production in in vitro systems, including cell cultures of $P$. corylifolia (Shinde et al. 2009a) and Pueraria tuberosa (Karwasara and Dixit 2012). However, a synergistic effect of other CW constituents, such as amino acids and growth regulators (Yong et al. 2009) cannot be excluded.

\section{Bioreactor experiments}

Selecting a proper bioreactor type and cultivation mode is crucial for obtaining productive in vitro system (Georgiev et al. 2009, 2013). Given that biomass cytodifferentiation is not required for isoflavone biosynthesis (Luczkiewicz 2008), attempts to establish biotechnological source of the above metabolites are based mainly on suspension cultures of legume plants. Their major advantage over the organised cultures, such as adventitious and hairy roots, is that they can be successfully grown in popular and commercially available stirred-tank bioreactors of laboratory and industrial scale. The above-mentioned systems have been used for the cultivation of cell suspensions of P. lobata (3.5 1 working volume, Chen and Li 2007), P. tuberosa (1.5 1 working volume, 
Sharma et al. 2009) and G. $\max (9.01$ working volume, Terrier et al. 2007), which are characterised by the presence of genistein and daidzein derivatives. The bioreactors were operated exclusively in batch or fed-batch mode, providing isoflavone yields higher or comparable to cultures grown in shake flasks. Although continuous culture seems to be a promising technological solution ensuring high productivity (Georgiev et al. 2013), few attempts to establish such a system for isoflavone production have been undertaken so far. The use of magnetofluidised bed bioreactor for the cultivation of soybean cells provided continuous biomass output; however, the achieved daidzein and genistein concentrations went down by ca. 50 and $80 \%$, respectively (Ames and Worden 1997).

Given the above, we decided to use a stirred-tank bioreactor and batch cultivation mode in the scale-up experiments. In order to prevent cell damage, the growth vessel was equipped with plate type impeller (Fig. 1) generating low shear stress (Georgiev et al. 2013). Air flow rate was set at $0.2 \mathrm{vvm}$ to provide $50 \%$ aeration, which was considered sufficient according to the previous reports (Terrier et al. 2007; Sharma et al. 2009) and also to prevent excessive foam formation. In order to counteract the loss of culture volume during the 40-day culture period, the bioreactor was equipped with air humidifier (Drechsel-type washing bottle) and outlet PTFE filter (Fig. 1), which effectively prevented medium evaporation and moisture escape.

In the presented study, the growth profile of bioreactorgrown cultures was similar to suspension cultivated in Erlenmeyer flasks, which was in accordance with other work on cell cultures of legume plants (Terrier et al. 2007; Sharma et al. 2009). Therefore, the examined biomass proved to be suitable for large-scale experiments, as it showed good resistance to mechanical stress generated by mechanical stirring. Foam formation, although generally unfavourable, did not decrease the biomass yield in the presented study.

Altered culture conditions clearly influenced the isoflavone production profiles. Two distinct production maxima were recorded instead of the one seen in isoflavone concentration curves characteristic for shake flask-grown cells (Figs. 3b-d and 4b-d). As previously suggested, the above phenomenon might be associated with de novo biosynthesis during the extended stationary phase. Despite the observed differences in isoflavone production kinetics, the calculated productivities, except for FG, were similar for both Erlenmeyer flasks and bioreactor system (Table 1).

In the modified experiment, the decision was made to grow the cultures without the presence of light as this strategy proved to improve isoflavone productivity in shake flask-grown cells (Fig. 2b-d). Moreover, the lack of light during cell cultivation is beneficial from the economic point of view, as it substantially reduces operational costs. A decision was also made to grow the cells in fed-batch mode, which is widely regarded as a simple and effective way of improving the productivity of secondary metabolites in in vitro systems (Georgiev et al. 2009, 2013). Given its stimulatory effect on biomass growth and isoflavone production (Fig. 3), CW was selected for fed-batch cultivation. Together with nutrient supplementation, antifoam agent was added to the growth medium in order to reduce wallgrowth and the formation of cell aggregates. Although no direct detrimental effects regarding isoflavone content and biomass concentration were observed in the current study, excessive foaming can seriously hamper bioreactor operation during scale-up experiments and continuous processes (Junker 2007).

The combined experimental approach resulted in faster biomass growth and altered isoflavone production kinetics in comparison to cell suspension grown in STB under continuous light (Fig. 4b-d). Foam formation was reduced without negative effect on cell vitality. The resulting CG, PG and FG productivities were higher than in the control group (STB without supplementation). Unfortunately, they remained lower than in the suspension cultures grown in shake flasks without the presence of light or supplemented with $\mathrm{CW}$. Bioreactor cultivation also seems less favourable for CG production (Table 1). The obtained results are difficult to compare with previous report as, to our knowledge, no plant in vitro systems oriented towards the production of methoxylated isoflavones have been developed so far. Among other potential sources of the compounds quoted are cell cultures of T. pratense, Maackia amurensis and A. membranaceus. However, studies on cell suspensions of $T$. pratense were limited to shake flasks and did not involve evaluation of CG, PG and FG levels (Kašparová et al. 2009). In vitro experiments on M. amurensis, on the other hand, are limited to callus cultures with $C G$ and FG content of ca. 50 and $80 \mathrm{mg} / 100 \mathrm{~g} \mathrm{DW}$, respectively (Fedoreyev et al. 2008). Isoflavone concentrations in $A$. membranaceus adventitious roots were either relatively low (ca. $1.3 \mathrm{mg} / 100 \mathrm{~g} \mathrm{DW}$ of CG; Thwe et al. 2012) or, as in the case of roots grown in balloon-type bioreactor, not evaluated at all (Wu et al. 2011). Hairy roots of the above species were also obtained and grown in air-lift bioreactor; however, their isoflavone content was not determined (Du et al. 2003).

Given the above, we conclude that the presented $C$. subternata in vitro system, together with the previously described downstream processing method (Kokotkiewicz et al. 2012b), can be used for the selective production of methoxy- (7-O- $\beta$-glucosides of calycosin and formononetin) and methylenedioxyisoflavones (pseudobaptigenin 7-O- $\beta$ glucoside) with expected therapeutic potential. Further work should be aimed at reducing the operational costs and improving the isoflavone productivity. The first goal can be achieved through the application of disposable bioreactors, which are becoming a popular alternative for the predominant glass and stainless steel fermentors. Their advantages 
include time and power savings, reduced contamination rates and low shear conditions for cell cultivation (Georgiev et al. 2013). The perspectives are promising, as disposable bioreactors were successfully used for maintaining isoflavoneproducing soybean cell suspension cultures. Isoflavone content in the Wave-and-Undertow and Slug Bubble (SB) bioreactors clearly exceeded the levels obtained in stirred-tank and, in the case of the $301 \mathrm{SB}$ system, also surpassed concentrations recorded for shake flask-grown cells (Terrier et al. 2007).

Apart from further optimisation of bioprocess parameters, isoflavone productivity of $C$. subternata cell cultures could possibly be increased by genetic transformation. It was shown that Agrobacterium rhizogenes rol oncogenes, apart from modulating plant growth and cell differentiation, are also potent activators of secondary metabolism, with rolC being the most studied in this regard. Moreover, activation of secondary metabolism by rol genes is stable over time, which is necessary for plant cell culture-based production of bioactive compounds (Zhou et al. 2011). The described strategy, involving incorporation of $\mathrm{rolC}$ gene via $A$. rhizogenes transformation, was also successfully applied in the case of $M$. amurensis in vitro cultures, which were previously mentioned as a potential source of methohylated isoflavones. As a result, 1.8, 1.6 and 3-fold increase in calycosin, pseudobaptigenin and formononetin concentrations was obtained, respectively (Grishchenko et al. 2013). Given that isoflavone biosynthesis can be activated by rol genes, the full potential of C. subternata cell cultures in terms of isoflavone production has yet to be revealed.

Acknowledgements This study was financially supported by grant no. NN302 041936 from the Polish Ministry of Education and Science.

Conflict of interest The authors declare that they have no conflict of interest.

Open Access This article is distributed under the terms of the Creative Commons Attribution License which permits any use, distribution, and reproduction in any medium, provided the original author(s) and the source are credited.

\section{References}

Ames TT, Worden RM (1997) Continuous production of daidzein and genistein from soybean in a magnetofluidized bed bioreactor. Biotechnol Prog 13:336-339

Barthe GA, Jourdan PS, McIntosh CA, Mansell RL (1987) Naringin and limonin production in callus cultures and regenerated shoots from Citrus sp. J Plant Physiol 127:55-65

Beerhues L, Berger U (1994) Xanthones in cell suspension cultures of two Centaurium species. Phytochemistry 35:1227-1231

Boonsnongcheep P, Korsangruang S, Soonthornchareonnon N, Chintapakorn Y, Saralamp P, Prathanturarug S (2010) Growth and isoflavonoid accumulation of Pueraria candollei var. candollei and P. candollei var. mirifica cell suspension cultures. Plant Cell Tissue Organ Cult 101:119-126

Chen G, Li L (2007) Nutrient consumption and production of isoflavones in bioreactor cultures of Pueraria lobata (Willd). J Environ Biol 28:321-326

Chen J, Liu L, Hou R, Shao Z, Wu Y, Chen X, Zhou L (2011) Calycosin promotes proliferation of estrogen receptor-positive cells via estrogen receptors and ERK1/2 activation in vitro and in vivo. Cancer Lett 308:144-151

Choi SI, Heo TR, Min B-H, Cui JH, Choi BH, Park SR (2007) Alleviation of osteoarthritis by calycosin-7- $O-\beta{ }_{-}$-glucopyranoside (CG) isolated from Astragali radix (AR) in rabbit osteoarthritis (OA) model. Osteoarthr Cartilage 15:1086-1092

De Beer D, Joubert E (2010) Development of HPLC method for Cyclopia subternata phenolic compound analysis and application to other Cyclopia spp. J Food Compos Anal 23:289-297

Du M, Wu XJ, Ding J, Hu ZB, White KN, Branford-White CJ (2003) Astragaloside IV and polysaccharide production by hairy roots of Astragalus membranaceus in bioreactors. Biotechnol Lett 25:1853-1856

Federici E, Touché A, Choquart S, Avanti O, Fay L, Offord E, Courtois D (2003) High isoflavone content and estrogenic activity of 25 year-old Glycine max tissue cultures. Phytochemistry 64:717-724

Fedoreyev SA, Bulgakov VP, Grishchenko OV, Veselova MV, Krivoschekova OE, Kulesh NI, Denisenko VA, Tchernoded GK, Zhuravlev YN (2008) Isoflavonoid composition of a callus culture of the relict tree Maackia amurensis Rupr. et Maxim. J Agric Food Chem 56:7023-7031

Georgiev MI, Eibl R, Zhong J-J (2013) Hosting the plant cells in vitro: recent trends in bioreactors. Appl Microbiol Biotechnol 97:3787-3800

Georgiev MI, Weber J, Maciuk A (2009) Bioprocessing of plant cell cultures for mass production of targeted compounds. Appl Microbiol Biotechnol 83:809-823

Goyal S, Ramawat KG (2008a) Ethrel treatment enhanced isoflavonoids accumulation in cell suspension cultures of Pueraria tuberosa, a woody legume. Acta Physiol Plant 30:849-853

Goyal S, Ramawat KG (2008b) Synergistic effect of morphactin on cytokinin-induced production of isoflavonoids in cell cultures of Pueraria tuberosa (Roxb. Ex. Willd.) DC. Plant Growth Regul 55:175-181

Grishchenko OV, Kiselev KV, Tchernoded GK, Fedoreyev SA, Veselova MV, Bulgakov VP, Zhuravlev YN (2013) The influence of the rolC gene on isoflavonoid production in callus cultures of Maackia amurensis. Plant Cell Tissue Organ Cult 113:429-435

Gueven A, Knorr D (2011) Isoflavonoid production by soy plant callus suspension culture. J Food Eng 103:237-243

Ha H, Lee HY, Lee J-H, Jung D, Choi J, Song K-Y, Jung HJ, Choi JS, Chang S-I, Kim C (2010) Formononetin prevents ovariectomyinduced bone loss in rats. Arch Pharm Res 33:626-632

Hashemi M, Behrangi N, Borna H, Akbarzadeh A (2012) Evaluating new targets of natural anticancer molecules through bioinformatics tools. J Proteomics Bioinform 5:50-53

Huh J-E, Seo D-M, Baek Y-H, Choi D-Y, Park D-S, Lee J-D (2010) Biphasic positive effect of formononetin on metabolic activity of human normal and osteoarthritic subchondral osteoblasts. Int Immunopharmacol 10:500-507

Junker B (2007) Foam and its mitigation in fermentation systems. Biotechnol Prog 23:767-784

Karwasara VS, Dixit VK (2012) Culture medium optimization for improved puerarin production by cell suspension cultures of Pueraria tuberosa (Roxb. ex Willd.) DC. In Vitro Cell Dev Biol - Plant 48:189-199

Kašparová M, Siatka T, Dušek J (2009) Production of isoflavonoids in the Trifolium pratense L. suspension culture. Ceska Slov Farm 58:66-69

Kokotkiewicz A, Luczkiewicz M, Hering A, Ochocka R, Gorynski K, Bucinski A, Sowinski P (2012a) Micropropagation of Cyclopia 
genistoides, an endemic South African plant of economic importance. Z Naturforsch $67 \mathrm{c}: 65-76$

Kokotkiewicz A, Luczkiewicz M, Sowinski P, Glod D, Gorynski K, Bucinski A (2012b) Isolation and structure elucidation of phenolic compounds from Cyclopia subternata Vogel (honeybush) intact plant and in vitro cultures. Food Chem 133:1373-1382

Kokotkiewicz A, Wnuk M, Bucinski A, Luczkiewicz M (2009) In vitro cultures of Cyclopia plants (honeybush) as a source of bioactive xanthones and flavanones. Z Naturforsch 64c:533-540

Li L, Zhang CR (2006) Production of puerarin and isoflavones in cell suspension cultures of Pueraria lobata (Willd.): effects of medium supplementation with casein hydrolysate and coconut milk. J Environ Biol 27:21-26

Luczkiewicz M, Cisowski W (2001) Optimization of the second phase of a two phase growth system for anthocyanin accumulation in callus cultures of Rudbeckia hirta. Plant Cell Tissue Organ Cult 65:57-68

Luczkiewicz M, Glod D (2005) Morphogenesis-dependent accumulation of phytoestrogens in Genista tinctoria in vitro cultures. Plant Sci 168:967-979

Luczkiewicz M, Kokotkiewicz A (2012) Elicitation and permeabilisation affect the accumulation and storage profile of phytoestrogens in high productive suspension cultures of Genista tinctoria. Acta Physiol Plant 34:1-16

Luczkiewicz MT (2008) Research into isoflavonoid phyto-oestrogens in plant cell cultures. In: Ramawat KG, Merillon JM (eds) Bioactive molecules and medicinal plants. Springer-Verlag, Berlin Heidelberg, pp 55-84

Mohamed MF, Read PE, Coyne DP (1992) Dark preconditioning, CPPU, and thidiazuron promote shoot organogenesis on seedling node explants of common and faba beans. J Amer Soc Hort Sci 117:668-672

Pan H, Fang C, Zhou T, Wang Q, Chen J (2007) Accumulation of calycosin and its 7-O- $\beta$-D-glucoside and related gene expression in seedlings of Astragalus membranaceus Bge. var. mongholicus (Bge.) Hsiao induced by low temperature stress. Plant Cell Rep 26:1111-1120

Pasqua G, Avato P, Monacelli B, Santamaria AR, Argentieri MP (2003) Metabolites in cell suspension cultures, calli, and in vitro regenerated organs of Hypericum perforatum cv. Topas. Plant Sci 165:977-982
Patisaul HB, Jefferson W (2010) The pros and cons of phytoestrogens. Front Neuroendocrinol 31:400-419

Sharma V, Goyal S, Ramawat KG (2009) Scale up production of isoflavonoids in cell suspension cultures of Pueraria tuberosa grown in shake flasks and bioreactor. Eng Life Sci 9:267-271

Shinde AN, Malpathak N, Fulzele DP (2009a) Studied enhancement strategies for phytoestrogens production in shake flasks by suspension culture of Psoralea corylifolia. Bioresour Technol 100:1833-1839

Shinde AN, Malpathak N, Fulzele DP (2009b) Optimized production of isoflavones in cell cultures of Psoralea corylifolia $\mathrm{L}$. using elicitation and precursor feeding. Biotechnol Bioprocess Eng 14:612-618

Terrier B, Courtois D, Hénault N, Cuvier A, Bastin M, Aknin A, Dubreuil J, Pétiard V (2007) Two new disposable bioreactors for plant cell culture: the wave and undertow bioreactor and the slug bubble bioreactor. Biotechnol Bioeng 96:914-923

Thwe AA, Mai NTT, Li X, Kim Y, Kim YB, MdR U, Kim YS, Bae H, Kim HH, Lee MY, Park SU (2012) Production of astragaloside and flavones from adventitious root cultures of Astragalus membranaceus var. mongholicus. Plant Omics J 5:466-470

Wu Q, Wang M, Simon JE (2003) Determination of isoflavones in red clover and related species by high-performance liquid chromatography combined with ultraviolet and mass spectrometric detection. J Chromatogr A 1016:195-209

Wu SQ, Lian ML, Gao R, Park SY, Piao XC (2011) Bioreactor application on adventitious root culture of Astragalus membranaceus. In Vitro Cell Dev Biol - Plant 47:719-724

Wu T, Bligh SWA, Gu L-H, Wang Z-T, Liu H-P, Cheng X-M, BranfordWhite CJ, Hu Z-B (2005) Simultaneous determination of six isoflavonoids in commercial Radix astragali by HPLC-UV. Fitoterapia 76:157-165

Yanagihara K, Ito A, Toge T, Numoto M (1993) Antiproliferative effects of isoflavones on human cancer cell lines established from the gastrointestinal tract. Cancer Res 53:5815-5821

Yong JWH, Ge L, Ng YF, Tan SN (2009) The chemical composition and biological properties of coconut (Cocos nucifera $\mathrm{L}$.) water. Molecules 14:5144-5164

Zhou M-L, Zhu X-M, Shao J-R, Tang Y-X, Wu Y-M (2011) Production and metabolic engineering of bioactive substances in plant hairy root culture. Appl Microbiol Biotechnol 90:1229-1239 